\title{
Use of controlled-release polymer to feed ammonium to Streptomyces clavuligerus cephalosporin fermentations in shake flasks
}

\section{Lübbe, A. L. Demain, and K. Bergman}

Fermentation Microbiology Laboratory, Department of Applied Biological Sciences, Massachusetts Institute of Technology, Cambridge, MA 02139, USA

Appl Microbiol Biotechnol (1985) 22:424-427

On p. 427 in Table 1 the column headings should read as follows:

$\begin{array}{lll}\mathrm{N} \text {-source } & \begin{array}{l}\text { Maximum volumetric titer } \\ \text { (Units cephalosporins } / \mathrm{ml} \text { ) }\end{array} & \begin{array}{l}\text { Maximum specific titer } \\ \text { (units cephalosporins } / \mathrm{mg} \text { DCW) }\end{array}\end{array}$ 